\title{
Attitude Tracking with Adaptive Rejection of Rate Gyro Disturbances
}

\author{
Pierluigi Pisu and Andrea Serrani
}

\begin{abstract}
The classical attitude control problem for a rigid body is revisited under the assumption that measurements of the angular rates obtained by means of rate gyros are corrupted by harmonic disturbances, a setup of importance in several aerospace applications. The paper extends previous methods developed to compensate bias in angular rate measurements by accounting for a more general class of disturbances, and by allowing uncertainty in the inertial parameters. By resorting to adaptive observers designed on the basis of the internal model principle, it is shown how converging estimates of the angular velocity can be obtained, and used effectively in a passivity-based certainty-equivalence controller yielding global convergence within the chosen parametrization of the group of rotations. Since a persistence of excitation condition is not required for the convergence of the state estimates, only an upper bound on the number of distinct harmonic components of the disturbance is needed for the applicability of the method.
\end{abstract}

\section{PROBLEM DEFINITION}

Consider the rotational dynamics of a rigid body

$$
\begin{aligned}
\dot{R} & =R S(\omega) \\
J(\mu) \dot{\omega} & =S(J(\mu) \omega) \omega+u,
\end{aligned}
$$

with state $(R, \omega) \in S O(3) \times \mathbb{R}^{3}$, representing the orientation and angular velocity of a body-fixed frame with respect to an inertial frame, and control input $u \in \mathbb{R}^{3}$. The matrix $S(\cdot)$ denotes the skew-symmetric operator $S(v) w:=v \times w$, where $v, w \in \mathbb{R}^{3}$. The inertia matrix $J(\mu) \in \mathbb{R}^{3 \times 3}$ is assumed to depend continuously on a vector of unknown parameters $\mu$ ranging over a given compact set $\mathcal{K}_{\mu} \subset \mathbb{R}^{p}$. For obvious reasons, it is assumed that $J(\mu)=J^{T}(\mu)>0$ for all $\mu \in$ $\mathcal{K}_{\mu}$. The desired reference trajectory $\left(R_{d}, \omega_{d}\right) \in S O(3) \times \mathbb{R}^{3}$ for the orientation and angular velocity of the body-fixed frame of (1) is provided by a smooth autonomous system of the form

$$
\begin{aligned}
\dot{\varpi}_{d} & =s\left(\varpi_{d}\right) \\
\dot{R}_{d} & =R_{d} S\left(\omega_{d}\right) \\
\omega_{d} & =r\left(\varpi_{d}\right)
\end{aligned}
$$

with state $\left(\varpi_{d}, R_{d}\right)$ evolving on a compact invariant subset $\mathcal{K}_{\varpi} \times S O(3)$ of $\mathbb{R}^{n_{d}} \times S O(3)$. This setup, while obviously not the most general, encompasses many configurations

This work has been supported in part by NSF under Grant No. 0220180, and by AFOSR and AFRL/VA through the Collaborative Center of Control Science (Contract No. F33615-01-2-3154.)

P. Pisu is with the Department of Mechanical Engineering, Clemson University, Clemson, SC 29634.

A. Serrani is with the Department of Electrical and Computer Engineering, The Ohio State University, Columbus, $\mathrm{OH} 43210$. Corresponding author: A. Serrani, 205 Dreese Lab, 2015 Neil Ave., Columbus, OH 43210 - USA. Email: serrani@ece.osu.edu of importance in aerospace applications [1], and greatly simplify the analysis. The rotation matrix for the attitude error $R_{e}:=R_{d}^{T} R \in S O(3)$ satisfies the kinematic equation $\dot{R}_{e}=R_{e} S\left(\omega_{e}\right)$, where $\omega_{e}:=\omega-R_{e}^{T} \omega_{d}$ denotes the angular velocity error resolved in the body frame.

The classic attitude control problem [2] is loosely defined as that of finding a feedback control law such that all trajectories of the closed-loop system are bounded, and the tracking error satisfies $\left(R_{e}(t), \omega_{e}(t)\right) \rightarrow\left(I_{3}, 0\right)$ as $t \rightarrow \infty$, for any given reference trajectory in the considered family of solutions of (2), and for all $\mu \in \mathcal{K}_{\mu}$. In this paper, the problem in question is revisited under the assumption that measurements of the rotation matrix $R(t)$ are available, while measurements of $\omega(t)$ obtained by means of rate gyros are corrupted by additive harmonic noise. The considered setup arises frequently in the control of aerospace vehicles with significant aeroelastic effects [3], where structural vibrations are transmitted to the rate gyros through the coupling with the airframe, or in the attitude control of rigid of flexible satellites, where harmonic disturbance in the angular velocity measurements are produced by imbalance or mechanical defects in gyroscopes [4]-[6]. Dealing with uncertainties on the natural frequencies is a fundamental issue in applications to control of hypersonic vehicles, where the vibrational modes change in response to mass variation and unsteady heating effects [7].

Building upon the results of [8], in this study the disturbance is modeled as an exogenous signal containing a finite number of harmonics of unknown amplitude, phase and frequency. While the formulation of the problem falls in principle within the scope of output regulation theory, the occurrence of the disturbance at the sensor input poses unique challenges, as the error to be regulated is not directly available to the controller [9]. For the problem at issue, it will be shown first that a converging estimate of the angular velocity can be obtained using an observer endowed with a nonlinear adaptive internal model of the exogenous disturbance. The design of the adaptive observer extends (nontrivially) the approach proposed in [6] to the more general situation discussed here. A remarkable feature of our approach is that only an upper bound on the number of distinct harmonics of the disturbance is required for the implementation of the adaptive observer, since persistence of excitation of the regressor is not needed for the convergence of the state estimates. Then, it will be shown that the availability of converging estimates of the angular velocity suffices to obtain global tracking (with respect to the chosen parametrization of the attitude error in $S O(3)$ ) by means of a certainty-equivalence robust redesign of the adaptive attitude 
regulator of Egeland and Godhavn [10]. Since the design of the regulator is independent from that of the observer, the result yields a form of separation principle for attitude regulation that may be applicable to more general situations.

The paper is organized as follows: The disturbance model is briefly described in Section II, whereas the design of the adaptive observer and the certainty-equivalence controller are presented in Section III and Section IV, respectively. Simulation results are illustrated in Section V, followed by concluding remarks.

\section{Disturbance Model}

Consider again the rigid body dynamics (1), and assume that the measurements of the angular velocity $\omega$ are affected by an additive harmonic disturbance $d \in \mathbb{R}^{3}$, in such a way that the output to be used for feedback control is given by

$$
y=\left(R, \omega_{a}\right) \in S O(3) \times \mathbb{R}^{3}, \quad \omega_{a}:=\omega+d .
$$

Each component of $d$ is assumed to be given by a finite sum of sinusoidal signals of unknown constant amplitude, frequency, and phase

$$
d_{i}(t)=\sum_{j=1}^{p_{i}} a_{i j} \sin \left(\theta_{i j} t+\phi_{i j}\right), \quad 1 \leq i \leq 3 .
$$

The numbers $p_{i} \in \mathbb{N}, 1 \leq i \leq 3$, are regarded as upper bounds on the number of distinct harmonics in $d$, which implies that some $a_{i j}$ may be identically zero. The disturbance signal $d(t), t \geq 0$, is generated by the neutrally stable system

$$
\begin{aligned}
\dot{w} & =\Phi(\theta) w \\
\dot{\theta} & =0 \\
d & =\Gamma w
\end{aligned}
$$

with state $w \in \mathbb{R}^{2 p}$, output $d \in \mathbb{R}^{3}$, parameter vector $\theta=\operatorname{col}\left(\theta_{1}, \theta_{2}, \theta_{3}\right) \in \mathbb{R}^{p}$, and matrices $\Phi(\theta)=$ $\operatorname{diag}\left(\Phi_{1}\left(\theta_{1}\right), \ldots, \Phi_{3}\left(\theta_{3}\right)\right), \Gamma=\operatorname{diag}\left(\Gamma_{1}, \ldots, \Gamma_{3}\right)$, where the pairs $\left(\Phi_{i}\left(\theta_{i}\right), \Gamma_{i}\right) \in \mathbb{R}^{2 p_{i} \times 2 p_{i}} \times \mathbb{R}^{1 \times 2 p_{i}}$ are observable. The vectors $\theta_{i} \in \mathbb{R}^{p_{i}}$ collect the unknown frequencies $\theta_{i j}$, $j=1 \ldots p_{i}$ of the harmonic components of the disturbances, while the initial condition $w(0)$ contains the information about the unknown amplitudes $a_{i j}$ and phases $\phi_{i j}$. It is assumed that a bound on the amplitude and the frequency of the harmonics in $d$ is known:

Assumption 2.1: The trajectories of (4) evolve within a given compact invariant set $\mathcal{K}=\mathcal{K}_{w} \times \mathcal{K}_{\theta} \subset \mathbb{R}^{2 p} \times \mathbb{R}_{+}^{p}$.

While the actual realization of system (4) is immaterial for the purpose of generating the disturbance signal $d(\cdot)$, a certain realization, referred to as a canonical parametrization in [11], is more convenient for internal model design. The realization in question stems from the following result (see [1, Lemma 1.5.6]):

Lemma 2.2: Let $F \in \mathbb{R}^{n \times n}, G \in \mathbb{R}^{n \times 1}, \Phi \in \mathbb{R}^{n \times n}$, and $\Gamma \in \mathbb{R}^{1 \times n}$ be such that the pair $(F, G)$ is controllable, the pair $(\Phi, \Gamma)$ is observable, $\mathrm{F}$ is Hurwitz, and $\Phi$ has all eigenvalues on the imaginary axis. Then, the unique solution
$M \in \mathbb{R}^{n \times n}$ of the Sylvester equation $M \Phi-F M=G \Gamma$ is nonsingular.

The result of Lemma 2.2 is exploited as follows: for each $i=1 \ldots 3$, fix Hurwitz matrices $F_{i} \in \mathbb{R}^{2 p_{i} \times 2 p_{i}}$ and matrices $G_{i} \in \mathbb{R}^{2 p_{i} \times 1}$ in such a way that $\left(F_{i}, G_{i}\right)$ is controllable, and denote $M_{i}\left(\theta_{i}\right) \in \mathbb{R}^{2 p_{i} \times 2 p_{i}}$ the unique (unknown) nonsingular solution of the Sylvester equation $M_{i}\left(\theta_{i}\right) \Phi\left(\theta_{i}\right)-F_{i} M_{i}\left(\theta_{i}\right)=G_{i} \Gamma_{i}$. Let $M(\theta):=$ $\operatorname{diag}\left\{M_{1}\left(\theta_{1}\right), \ldots, M_{3}\left(\theta_{3}\right)\right\}$ and consider the transformation $\bar{w}:=M(\theta) w$. In the new set of coordinates, system (4) reads as

$$
\begin{aligned}
\dot{\bar{w}} & =(F+G \Psi(\theta)) \bar{w} \\
\dot{\theta} & =0 \\
d & =\Psi(\theta) \bar{w}
\end{aligned}
$$

where $F:=\operatorname{diag}\left\{F_{1}, \ldots, F_{3}\right\}, G:=\operatorname{diag}\left\{G_{1}, \ldots, G_{3}\right\}$, $\Psi(\theta):=\operatorname{diag}\left\{\Psi_{1}\left(\theta_{1}\right), \ldots, \Psi_{3}\left(\theta_{3}\right)\right\}$, and $\Psi_{i}\left(\theta_{i}\right) \quad:=$ $\Gamma_{i} M_{i}^{-1}\left(\theta_{i}\right)$.

Remark 2.1: By using a technique similar to the one proposed in [11], the disturbance models (4) and (5) can be modified to include a constant bias in the signal $d$, thus incorporating the setup considered in [6]. Since such an extension does not lead to a substantial modification of the control algorithm, it has been omitted for simplicity.

\section{AdAptive Attitude Observer}

Following [12], an observer for the attitude dynamics (1) endowed with the measurement equation (3) can be defined as the system

$$
\dot{\hat{R}}=\hat{R} S(\hat{\Omega}(y))
$$

evolving on $S O(3)$, where the observer angular velocity $\hat{\Omega}=$ $\hat{\Omega}(y)$ resolved in the observer frame is a function (static or dynamic) of the measured variables. The observer error dynamics for (6) and (1) is given by

$$
\dot{\tilde{R}}=\tilde{R} S(\tilde{\omega})
$$

where $\tilde{R}:=\hat{R}^{T} R$ and $\tilde{\omega}:=\omega-\tilde{R}^{T} \hat{\Omega}$ denote respectively the observer error rotation matrix and its angular velocity resolved in the body frame. For the problem under investigation, the observer should incorporate an adaptive internal model of the disturbance of the form

$$
\begin{aligned}
\dot{\zeta} & =\varphi(\zeta, \hat{\psi}, y) \\
\dot{\hat{\psi}} & =\phi(\zeta, y) \\
\hat{\Omega} & =\hat{\Omega}(\zeta, \hat{\psi}, y)
\end{aligned}
$$

with state $\zeta \in \mathbb{R}^{n_{\zeta}}$, tunable parameter vector $\hat{\psi} \in \mathbb{R}^{n_{\psi}}$, and input given by the available measurement $y$. The role of (8) is to reconstruct the disturbance $d$, and to provide asymptotic convergence of $\tilde{R}(t)$ to the identity matrix. While the structure of the observer is conceptually similar to the one employed in [6], the higher complexity of (8) is required to handle the uncertainty on the frequencies of the harmonic components of the disturbance.

To avoid manipulating rotation matrices directly, a minimal parametrization of $S O(3)$ will be employed in the 
design of the internal model. Let $(\tilde{\eta}, \tilde{\varepsilon}) \in \mathbb{S}^{4}$ denote the Euler parameters of $\tilde{R}$. The Modified Rodriguez Parameters (MRP), defined as $\tilde{\sigma}:=\tilde{\varepsilon} /(1+\tilde{\eta})$, offer a singularity-free representation of rotations in the range $[0,2 \pi)$ (see [13]). As a result, the MRP can be used as a global parametrization of the orientation error, once one identifies the initial conditions $(\tilde{\eta}(0), \tilde{\varepsilon}(0))=( \pm 1,0)$. As a matter of fact, for a given initial condition $\tilde{\sigma}(0) \in \mathbb{R}^{3}$ and a given locally essentially bounded input $\tilde{\omega}(t), t \geq 0$, boundedness of the trajectories $\tilde{\sigma}(t), t \geq 0$, of the propagation equation

$$
\dot{\tilde{\sigma}}=\frac{1}{2} E(\tilde{\sigma}) \tilde{\omega},
$$

where $E(\tilde{\sigma}):=\frac{1}{2}\left[1-\tilde{\sigma}^{T} \tilde{\sigma}\right] I_{3}+S(\tilde{\sigma})+S(\tilde{\sigma})^{2}$, implies that the corresponding rotation matrix

$$
\tilde{R}(\tilde{\sigma})=I_{3}+\frac{8}{\left(1+\tilde{\sigma}^{T} \tilde{\sigma}\right)^{2}} S(\tilde{\sigma})\left[S(\tilde{\sigma})+\frac{1}{2}\left(1-\tilde{\sigma}^{T} \tilde{\sigma}\right) I_{3}\right]
$$

describes rotations of instantaneous angles within $[0,2 \pi)$. With this in mind, consider the following expression for the nonlinear adaptive internal model

$$
\begin{aligned}
\dot{\xi} & =[F+2 G E(\tilde{\sigma}) \hat{\Psi}] \xi+N(\tilde{\sigma}) \\
\dot{\hat{\Psi}}_{i} & =-\gamma \tilde{\sigma}_{i} \xi_{i}^{T}, \quad 1 \leq i \leq 3 \\
\hat{\Omega} & =\tilde{R}(\tilde{\sigma})\left[\omega_{a}-\hat{\Psi} \xi+\kappa(\tilde{\sigma})\right]
\end{aligned}
$$

with state and tunable parameter vectors given respectively by $\xi=\operatorname{col}\left(\xi_{1}, \xi_{2}, \xi_{3}\right), \xi_{i} \in \mathbb{R}^{2 p_{i}}$, and $\hat{\Psi}_{i} \in \mathbb{R}^{2 p_{i}}, 1 \leq i \leq 3$, input $y=\operatorname{col}\left(\tilde{\sigma}, \omega_{a}\right)$, and adaptation gain $\gamma>0$. The interconnection term $N(\tilde{\sigma})$ and the stabilizing term $\kappa(\tilde{\sigma})$ in (10) are chosen respectively as

$$
\begin{aligned}
N(\tilde{\sigma}) & =-2 G E(\tilde{\sigma}) \kappa(\tilde{\sigma})-4 F G \tilde{\sigma} \\
\kappa(\tilde{\sigma}) & =k_{1} \tilde{\sigma}+k_{2}\|\tilde{\sigma}\|^{2} \tilde{\sigma}
\end{aligned}
$$

where $k_{1}, k_{2}>0$ are gain parameters. The motivation behind this choice will be given shortly. The matrix $\hat{\Psi}$ has the same structure as $\Psi$ in (5). Augmenting (10) with the equations of the observation error (9) and the exosystem (4) yields (after some algebra) the system

$$
\begin{aligned}
\dot{w} & =\Phi(\theta) w \\
\dot{\theta} & =0 \\
\dot{\xi} & =[F+2 G E(\tilde{\sigma}) \hat{\Psi}] \xi+N(\tilde{\sigma}) \\
\dot{\hat{\Psi}}_{i} & =-\gamma \tilde{\sigma}_{i} \xi_{i}^{T}, \quad 1 \leq i \leq 3 \\
\dot{\tilde{\sigma}} & =\frac{1}{2} E(\tilde{\sigma})[\hat{\Psi} \xi-\kappa(\tilde{\sigma})-\Gamma w]
\end{aligned}
$$

where the observation error for the angular rates reads as $\tilde{\omega}=\hat{\Psi} \xi-\kappa(\tilde{\sigma})-\Gamma w$. It is worth noting that system (12) is autonomous.

Proposition 3.1: Fix an arbitrary compact set $\mathcal{K}$ as in Assumption 2.1 for the initial conditions $(w(0), \theta(0))$. Then, there exist $k_{1}^{\star}>0$ and $k_{2}^{\star}>0$, depending only on $\mathcal{K}$, such that for any $k_{1}>k_{1}^{\star}$, any $k_{2}>k_{2}^{\star}$, and any $\gamma>0$, the solutions of (12) originating from any initial condition $\tilde{\sigma}(0) \in \mathbb{R}^{3}, \xi(0) \in \mathbb{R}^{2 p}$, and $\hat{\Psi}_{i}(0) \in \mathbb{R}^{2 p_{i}}, 1 \leq i \leq 3$, are bounded, and satisfy $\lim _{t \rightarrow \infty} \tilde{\sigma}(t)=0, \lim _{t \rightarrow \infty} \tilde{\omega}(t)=0$, and $\lim _{t \rightarrow \infty} \dot{\tilde{\omega}}(t)=0$.
Proof: Performing the change of coordinates $\chi:=\xi-$ $M(\theta) w-4 G \tilde{\sigma}$, the following expression is obtained in place of the $\xi$-dynamics:

$$
\begin{gathered}
\dot{\chi}=[F+2 G E(\tilde{\sigma}) \hat{\Psi}] \xi+N(\tilde{\sigma})-M(\theta) \Phi(\theta) w \\
-2 G E(\tilde{\sigma})[\hat{\Psi} \xi-\kappa(\tilde{\sigma})-\Gamma w] \\
=\quad F \chi+N(\tilde{\sigma})+2 G E(\tilde{\sigma}) \kappa(\tilde{\sigma})+4 F G \tilde{\sigma} \\
+2 G E(\tilde{\sigma}) \Gamma w-\Gamma w .
\end{gathered}
$$

The choice for $N(\tilde{\sigma})$ given in (11) decouples the $\chi$-from the $\tilde{\sigma}$-dynamics when $w=0$, and, most importantly, renders the $\chi$-dynamics independent from the stabilizing control $\kappa(\tilde{\sigma})$. Changing coordinates as $\tilde{\Psi}_{i}:=\hat{\Psi}_{i}-\Psi_{i}(\theta)$, system (12) can be viewed as the cascade interconnection of system (4) and system

$$
\begin{aligned}
\dot{\chi} & =F \chi+\Delta_{1}(\tilde{\sigma}, w) \\
\dot{\tilde{\Psi}}_{i} & =-\gamma \tilde{\sigma}_{i} \xi_{i}^{T}, \quad 1 \leq i \leq 3 \\
\dot{\tilde{\sigma}} & =\frac{1}{2} E(\tilde{\sigma})[4 \Psi(\theta) G \tilde{\sigma}-\kappa(\tilde{\sigma})+\Psi(\theta) \chi+\tilde{\Psi} \xi]
\end{aligned}
$$

where $\Delta_{1}(\tilde{\sigma}, w):=G\left[2 E(\tilde{\sigma})-I_{3}\right] \Gamma w, \xi=\chi+$ $M(\theta) w+4 G \tilde{\sigma}$, and $\tilde{\Psi}:=\operatorname{diag}\left(\tilde{\Psi}_{1}, \ldots, \tilde{\Psi}_{3}\right)$. Let $P=$ $\operatorname{diag}\left(P_{1}, \ldots, P_{3}\right)$, where each $P_{i}=P_{i}^{T}, 1 \leq i \leq 3$, solves the Lyapunov equations $P_{i} F_{i}+F_{i}^{T} P_{i}=-I$, and consider for system (13) the Lyapunov-like function candidate

$$
V(\chi, \tilde{\sigma}, \tilde{\Psi})=\chi^{T} P \chi+2 \ln \left(1+\tilde{\sigma}^{T} \tilde{\sigma}\right)+\frac{1}{2 \gamma} \sum_{i=1}^{3} \tilde{\Psi}_{i} \tilde{\Psi}_{i}^{T},
$$

whose Lie derivative along the vector field of system (13) reads as

$$
\dot{V}=-\chi^{T} \chi+2 \chi^{T} P \Delta_{1}(\tilde{\sigma}, w)+\tilde{\sigma}^{T}\left[4 \Psi(\theta) G \tilde{\sigma}-\tilde{\sigma}^{T} \kappa(\tilde{\sigma})\right] .
$$

The definition of $E(\tilde{\sigma})$ and continuity of the entries of $\Psi(\theta)$ with respect to $\theta$ imply that there exist positive numbers $\delta_{i}$, $i=1, \ldots, 3$, which depend only on the set $\mathcal{K}$, such that for all $(w, \theta) \in \mathcal{K}$

$$
\left\|P \Delta_{1}(\tilde{\sigma}, w)\right\|^{2} \leq \delta_{1}\|\tilde{\sigma}\|^{2}+\delta_{2}\|\tilde{\sigma}\|^{4},\|\Psi(\theta) G\| \leq \delta_{3} .
$$

The above bounds motivate the choice of the stabilizing control $\kappa(\tilde{\sigma})$ in (11). Finally, application of Young's inequality in the form $|a b| \leq \frac{1}{2} a^{2}+\frac{1}{2} b^{2}$ yields

$$
\dot{V} \leq-\frac{1}{2}\|\chi\|^{2}+\left(\delta_{1}+4 \delta_{3}-k_{1}\right)\|\tilde{\sigma}\|^{2}+\left(\delta_{2}-k_{2}\right)\|\tilde{\sigma}\|^{4} .
$$

By choosing $k_{1}>k_{1}^{\star}:=\delta_{1}+4 \delta_{3}$ and $k_{2}>k_{2}^{\star}:=\delta_{2}$, the derivative of $V$ along the vector field of system (13) is rendered negative semi-definite for all $(w, \theta) \in \mathcal{K}$. This implies that the equilibrium at the origin of system (13) is uniformly stable in the sense of Lyapunov, and that trajectories of the cascade system (4)-(13) originating from $\mathcal{K} \times$ $\mathbb{R}^{4 p+3}$ are bounded. Keeping in mind that the cascade (4)(13) is an autonomous system, application of La Salle's invariance principle implies that all trajectories originating from $\mathcal{K} \times \mathbb{R}^{4 p+3}$ converge to the largest invariant set $\mathcal{M}$ contained in $\mathcal{K} \times\{\dot{V}=0\}$. This yields $\lim _{t \rightarrow \infty} \tilde{\sigma}(t)=$ 0 , and thus $\lim _{t \rightarrow \infty} \tilde{R}(t)=I_{3}$. Furthermore, on the set $\mathcal{M}$ the trajectories $\tilde{\Psi}_{i}(t), t \geq 0$, are necessarily constant. 
Let $\left(w_{0}, \theta\right) \in \mathcal{K}$ denote the initial condition for (4), and let $\left(w\left(t, w_{0}\right), \theta\right), t \geq 0$, be the corresponding trajectory. Similarly to the proof of La Salle's principle, it is seen that invariance of $\mathcal{M}$ implies that

$$
\tilde{\Psi}^{\circ} M(\theta) w\left(t, w_{0}\right)=0 \quad \forall t \geq 0, \quad \forall \tilde{\Psi}^{\circ} \in \mathcal{M}
$$

and thus $\lim _{t \rightarrow \infty} \tilde{\Psi}(t) M(\theta) w\left(t, w_{0}\right)=0$. Recalling that $\tilde{\omega}=\tilde{\Psi} M(\theta) w+\hat{\Psi}[\chi+4 G \tilde{\sigma}]$, it is readily seen that $\lim _{t \rightarrow \infty} \tilde{\omega}(t)=0$. To establish that $\dot{\tilde{\omega}}(t)$ vanishes asymptotically, it suffices to notice that $\dot{\chi}(t), \dot{\tilde{\Psi}}(t)$ and $\dot{\tilde{\sigma}}(t)$ vanish on $\mathcal{M}$, and that (15) implies $\tilde{\Psi}^{\circ} M(\theta) \dot{w}\left(t, w_{0}\right)=0$ for all $t \geq 0$.

Remark 3.1: It is worth noting that the convergence of the observation error $(\tilde{\sigma}(t), \tilde{\omega}(t))$ to the origin does not require persistence of excitation $(\mathrm{PE})$ of the "regressor" $\xi(t)$ employed in the update law for $\hat{\Psi}_{i}$. Since PE of $\xi(t)$ is related to the richness of the exogenous signal $d(t)$, the result of Proposition 3.1 implies that knowledge of an upper bound on the number of distinct harmonics in $d$ is a sufficient condition for observer design. On the other hand, the estimation error $\tilde{\Psi}(t)$, which is related to the estimation of the unknown vector $\theta$, in general does not converge to the origin, rather to a subspace satisfying (15) at each point. In case the signal $w(t)$ - equivalently, $\xi(t)$ - is persistently exciting, standard arguments can be invoked to show that equation (15) yields $\tilde{\Psi}^{\circ}=0$.

\section{TRACKING CONTROLLER}

The design of the measurement-feedback controller is completed by a tracking controller, designed on the basis of the separation principle. The controller is a certaintyequivalence version of the standard passivity-based adaptive attitude regulator of [10], where state-feedback is replaced by feedback from the estimated angular velocity, and a robust redesign of the update law is employed. While it is customary to retain the original coordinates of the plant for stability analysis, for the problem at issue it is more advantageous to perform the analysis entirely in the observer coordinate system, and regard the observation error as a converging disturbance.

Consider the observed attitude tracking error $\hat{R}_{e}:=R_{d}^{T} \hat{R}$, with corresponding observed angular velocity error $\hat{\Omega}_{e}:=$ $\hat{\Omega}-\hat{R}_{e}^{T} \omega_{d}$ resolved in the observer frame, satisfying the equation $\dot{\hat{R}}_{e}=\hat{R}_{e} S\left(\hat{\Omega}_{e}\right)$. Let $\hat{\sigma}_{e} \in \mathbb{R}^{3}$ denote the MRP of $\hat{R}_{e}$, with propagation equation $\dot{\hat{\sigma}}_{e}=\frac{1}{2} E\left(\hat{\sigma}_{e}\right) \hat{\Omega}_{e}$. Define the augmented reference vector in the observer frame

$$
\hat{z}_{d}:=\hat{R}_{e}^{T} \omega_{d}-\hat{\sigma}_{e}
$$

and let $\hat{z}:=\tilde{R}^{T}\left(\hat{\Omega}-\hat{z}_{d}\right)=\hat{\omega}-\tilde{R}^{T} \hat{z}_{d}$ be the corresponding tracking error expressed in the body-fixed frame, where for notational convenience we have denoted by $\hat{\omega}=\tilde{R}^{T} \hat{\Omega}$ the estimated angular velocity resolved in the body frame. Then, the angular velocity of the rigid body can be expressed in terms of the new coordinates $\hat{z}$ as

$$
\omega=\hat{z}+\tilde{R}^{T} \hat{z}_{d}+\tilde{\omega}=\hat{\omega}+\tilde{\omega} .
$$

Substituting (17) into the bottom equation in (1), yields

$$
\begin{aligned}
J \dot{\hat{z}}+J \dot{\tilde{R}}^{T} \hat{z}_{d}+J \tilde{R}^{T} \dot{\hat{z}}_{d}+J \dot{\tilde{\omega}}= & S\left(J \hat{z}+J \tilde{R}^{T} \hat{z}_{d}+J \tilde{\omega}\right) \times \\
& {\left[\hat{z}+\tilde{R}^{T} \hat{z}_{d}+\tilde{\omega}\right]+u, }
\end{aligned}
$$

where the dependence of $J$ on $\mu$ has been suppressed to simplify the notation. By expanding the left side of the above equation, one obtains

$$
\begin{aligned}
J \dot{\tilde{R}}^{T} \hat{z}_{d} & =-J S(\tilde{\omega}) \tilde{R}^{T} \hat{z}_{d} \\
J \tilde{R}^{T} \dot{\hat{z}}_{d} & =J \tilde{R}^{T}\left[\dot{\hat{R}}_{e}^{T} \omega_{d}+\hat{R}_{e}^{T} \dot{\omega}_{d}-\frac{1}{2} E\left(\hat{\sigma}_{e}\right) \hat{\Omega}_{e}\right] \\
& =J \tilde{R}^{T}\left[-S\left(\hat{\Omega}_{e}\right) \hat{R}_{e}^{T} \omega_{d}+\hat{R}_{e}^{T} \dot{\omega}_{d}-\frac{1}{2} E\left(\hat{\sigma}_{e}\right) \hat{\Omega}_{e}\right] .
\end{aligned}
$$

While the term in (18) vanishes with the observation error, the term in (19) must be compensated by means of a feedforward action. Without loss of generality, assume that $J(\mu)$ depends linearly on $\mu$, and write

$$
J(\mu) \tilde{R}^{T} \dot{\hat{z}}_{d}-S\left(J(\mu) \hat{z}+J(\mu) \tilde{R}^{T} \hat{z}_{d}\right) \tilde{R}^{T} \hat{z}_{d}=Y\left(\tilde{R}, \boldsymbol{\omega}_{d}, \hat{\boldsymbol{x}}\right) \mu
$$

where the regressor $Y\left(\tilde{R}, \boldsymbol{\omega}_{d}, \hat{\boldsymbol{x}}\right)$ is a known function of the state $\hat{\boldsymbol{x}}:=\operatorname{col}\left(\hat{\sigma}_{e}, \hat{z}\right)$ and the available exogenous signals $\tilde{R}(t)$ and $\omega_{d}(t):=\operatorname{col}\left(\omega_{d}(t), \dot{\omega}_{d}(t)\right), t \geq 0$. The control input is chosen as the combination of a feedback and an adaptive feedforward control

$$
\begin{aligned}
\dot{\hat{\mu}} & =-\nu \operatorname{Proj}\left(Y\left(\tilde{R}, \boldsymbol{\omega}_{d}, \hat{\boldsymbol{x}}\right) \hat{z}\right) \\
u & =-\tilde{R}^{T} \hat{\sigma}_{e}-k_{3} \hat{z}+Y\left(\tilde{R}, \boldsymbol{\omega}_{d}, \hat{\boldsymbol{x}}\right) \hat{\mu}
\end{aligned}
$$

where $\nu>0, k_{3}>0$ are gain parameters, and $\operatorname{Proj}(\cdot)$ denotes the smooth projection onto a convex set $\mathcal{P} \supset \mathcal{K}_{\mu}$, as defined in [14, App. E]. This yields the system

$$
\begin{aligned}
\dot{\hat{\sigma}}_{e}= & \frac{1}{2} E\left(\hat{\sigma}_{e}\right)\left[\tilde{R}(t) \hat{z}-\hat{\sigma}_{e}\right] \\
J(\mu) \dot{\hat{z}}= & S(J(\mu) \hat{\omega}) \hat{z}-\tilde{R}^{T}(t) \hat{\sigma}_{e}-k_{3} \hat{z}+Y\left(\tilde{R}, \boldsymbol{\omega}_{d}, \hat{\boldsymbol{x}}\right) \tilde{\mu} \\
& \quad+\Delta_{2}(\hat{\boldsymbol{x}}, \tilde{R}, \tilde{\boldsymbol{\omega}}, \mu) \\
\dot{\tilde{\mu}}= & -\nu \operatorname{Proj}\left(Y\left(\tilde{R}, \boldsymbol{\omega}_{d}, \hat{\boldsymbol{x}}\right) \hat{z}\right)
\end{aligned}
$$

where $\tilde{\mu}:=\hat{\mu}-\mu, \tilde{\omega}:=\operatorname{col}(\tilde{\omega}, \dot{\tilde{\omega}})$, and

$$
\begin{aligned}
& \Delta_{2}(\hat{\boldsymbol{x}}, \tilde{R}, \tilde{\boldsymbol{\omega}}, \mu) \quad:=\left[J S\left(\tilde{R}^{T} \hat{z}_{d}\right)+S(J \hat{\omega})-S(\hat{\omega}) J\right] \tilde{\omega} \\
& +S(J \tilde{\omega}) \tilde{\omega}-J \dot{\tilde{\omega}}
\end{aligned}
$$

is a perturbation which vanishes at $\tilde{\boldsymbol{\omega}}=0$. The main result of the section is the following:

Proposition 4.1: Consider the closed-loop system given by the cascade interconnection of the controlled system (21) with the observer dynamics (12) and the reference generator (2). Let the assumptions of Proposition 3.1 be satisfied, and let a compact set $\mathcal{D}_{d} \subset \mathbb{R}^{6}$ be defined such that $\boldsymbol{\omega}_{d} \in \mathcal{D}_{d}$ for all $\varpi_{d} \in \mathcal{K}_{\varpi}$. Fix arbitrary initial conditions $(w(0), \theta) \in \mathcal{K}, \xi(0) \in \mathbb{R}^{2 p}, \hat{\Psi}_{i}(0) \in \mathbb{R}^{2 p_{i}}, 1 \leq i \leq 3$, $\tilde{\sigma}(0) \in \mathbb{R}^{3}$, and $\varpi_{d}(0) \in \mathcal{K}_{\varpi}$. Then, for any $k_{3}>0$ and $\nu>0$, the solution of (21) originating from any initial condition $\left(\hat{\boldsymbol{x}}(0), \hat{\mu}(0) \in \mathbb{R}^{6} \times \mathcal{P}\right.$, any $\mu \in \mathcal{K}_{\mu}$, and forced by the output $(\tilde{R}, \tilde{\boldsymbol{\omega}})$ and $\left(R_{d}, \boldsymbol{\omega}_{d}\right)$ of systems (12) and (2), respectively, is bounded and satisfies $\lim _{t \rightarrow \infty} \hat{\boldsymbol{x}}(t)=0$.

Proof: We proceed by establishing first that (21) is forward complete for all forcing signals considered here. 
System (21) is then shown to possess a bounded-input bounded-state property, without restriction on the initial state, and nonzero restriction on the disturbance input $\tilde{\boldsymbol{\omega}}$. Boundedness of all trajectories follows from the fact that restrictions on $\tilde{\boldsymbol{\omega}}$ are fulfilled in finite time. The proof is then completed by using standard arguments. Consider the positive definite and radially unbounded Lyapunov function candidate $W(\hat{\boldsymbol{x}}, \tilde{\mu})=2 \ln \left(1+\hat{\sigma}_{e}^{T} \hat{\sigma}_{e}\right)+\frac{1}{2} \hat{z}^{T} J(\mu) \hat{z}+\frac{1}{2 \nu} \tilde{\mu}^{T} \tilde{\mu}$ yielding, along trajectories of (21),

$$
\dot{W}(\hat{\boldsymbol{x}}, \tilde{\mu})=-\hat{\sigma}_{e}^{T} \hat{\sigma}_{e}-k_{3} \hat{z}^{T} \hat{z}+\hat{z}^{T} \Delta_{2}(\hat{\boldsymbol{x}}, \tilde{R}, \tilde{\boldsymbol{\omega}}, \mu) .
$$

Once the compact set $\mathcal{D}_{d}$ has been fixed, from the definition of $\Delta_{2}$ and $\hat{z}_{d}$ in (22) and (16) respectively, it follows that there exist positive numbers $\lambda_{i}, 0 \leq i \leq 4$, such that

$$
\begin{aligned}
& \left\|\Delta_{2}(\hat{\boldsymbol{x}}, \tilde{R}, \tilde{\boldsymbol{\omega}}, \mu)\right\| \leq\left(\lambda_{0}+\lambda_{1}\left\|\hat{\sigma}_{e}\right\|+\lambda_{2}\|\hat{z}\|\right)\|\tilde{\omega}\| \\
& +\lambda_{3}\|\tilde{\omega}\|^{2}+\lambda_{4}\|\dot{\tilde{\omega}}\|
\end{aligned}
$$

for all $t \geq 0$ and all $\mu \in \mathcal{K}_{\mu}$. As a result, for any $\tilde{\boldsymbol{\omega}}(\cdot) \in \mathcal{L}_{\infty}$, there exist numbers $\bar{\lambda}_{i}>0$ (depending on $\|\tilde{\omega}\|_{\infty}$ ) such that

$$
\left\|\Delta_{2}(\hat{\boldsymbol{x}}, \tilde{R}(t), \tilde{\boldsymbol{\omega}}(t), \mu)\right\| \leq \bar{\lambda}_{0}+\bar{\lambda}_{1}\left\|\hat{\sigma}_{e}\right\|+\bar{\lambda}_{2}\|\hat{z}\|
$$

for all $t \geq 0$ and all $\mu \in \mathcal{K}_{\mu}$. A simple application of Young's inequality shows that the derivative of $W(\cdot)$ along trajectories of (21) satisfies

$$
\dot{W}(\hat{\boldsymbol{x}}, \tilde{\mu}) \leq-\frac{1}{2}\left\|\hat{\sigma}_{e}\right\|^{2}+\bar{k}\|\hat{z}\|^{2}+\frac{1}{2} \bar{\lambda}_{0}^{2},
$$

where $\bar{k}:=\frac{1}{2}+\frac{1}{2} \bar{\lambda}_{1}^{2}+\bar{\lambda}_{2}-k_{3}$. Since $\ln \left(1+\sigma^{T} \sigma\right) \leq\|\sigma\|^{2}$ for all $\sigma \in \mathbb{R}^{3}$, it follows that

$\dot{W}(\hat{\boldsymbol{x}}, \tilde{\mu}) \leq \frac{1}{2} \ln \left(1+\hat{\sigma}_{e}^{T} \hat{\sigma}_{e}\right)+\bar{k}\|\hat{z}\|^{2}+\frac{1}{2} \bar{\mu}_{0}^{2} \leq \alpha W(\hat{\boldsymbol{x}}, \tilde{\mu})+\beta$

for some $\alpha>0, \beta=\frac{1}{2} \bar{\lambda}_{0}^{2}$, and for all $(\hat{\boldsymbol{x}}, \tilde{\mu}) \in \mathbb{R}^{6} \times \mathbb{R}^{p}$. Fix an arbitrary initial condition $(\hat{\boldsymbol{x}}(0), \hat{\mu}(0)) \in \mathbb{R}^{6} \times \mathcal{P}$, and let $\left[0, T_{\max }\right)$ denote the maximal interval of existence and uniqueness of the corresponding (forced) solution $(\hat{\boldsymbol{x}}(\cdot), \tilde{\mu}(\cdot))$ of $(21)$. Recall that, due to the properties of the projection operator, $\tilde{\mu}(t)$ is bounded over $\left[0, T_{\max }\right)$. Furthermore, along the given solution, the estimate

$$
W(\hat{\boldsymbol{x}}(t), \tilde{\mu}(t)) \leq \mathrm{e}^{\alpha t} W(\hat{\boldsymbol{x}}(0), \tilde{\mu}(0))+\frac{\beta}{\alpha}\left(\mathrm{e}^{\alpha t}-1\right)
$$

holds for all $t \in\left[0, T_{\max }\right)$, showing that $\hat{\boldsymbol{x}}(t)$ is bounded as well over $\left[0, T_{\max }\right)$, and thus $T_{\max }=+\infty$. Using again the bound (23), $\dot{W}(\hat{\boldsymbol{x}}, \tilde{\mu})$ can be estimated as

$$
\begin{aligned}
\dot{W} \leq- & \left\|\hat{\sigma}_{e}\right\|^{2}-\left(k_{3}-\lambda_{2}\|\tilde{\omega}\|\right)\|\hat{z}\|^{2}+\lambda_{1}\left\|\hat{\sigma}_{e}\right\|\|\hat{z}\|\|\tilde{\omega}\| \\
& +\left(\lambda_{0}\|\tilde{\omega}\|+\lambda_{3}\|\tilde{\omega}\|^{2}+\lambda_{4}\|\dot{\tilde{\omega}}\|\right)\|\hat{z}\|
\end{aligned}
$$

for all $\boldsymbol{\omega}_{d} \in \mathcal{D}_{d}$ and all $\mu \in \mathcal{K}_{\mu}$. An easy computation shows that for any given $k_{3}>0$ the trajectories of (21) are bounded if the restriction $\|\tilde{\omega}\|_{\infty}<2\left(\sqrt{\lambda_{2}^{2}+2 k_{3} \lambda_{1}^{2}}-\lambda_{2}\right) / \lambda_{1}^{2}$ is satisfied. Since $\tilde{\boldsymbol{\omega}}(t) \rightarrow 0$, the restriction is fulfilled in finite time, and thus the solution of system (21) is bounded. This in turn implies that any solution of the autonomous system given by the cascade interconnection, with initial condition in the considered set, is bounded, and thus its $\omega$-limit set is non-empty, compact and invariant. Denote by $\Omega_{+}$the $\omega$ limit set of an arbitrary such trajectory, and notice that on $\Omega_{+}$ necessarily $\tilde{\boldsymbol{\omega}}=0$. From (24), following standard arguments as in the proof of La Salle's principle, one concludes that $W$ is constant on $\Omega_{+}$, and thus $\hat{\boldsymbol{x}}=0$.

\section{Simulation Results}

Nonlinear simulations have been performed using a rigid body model with $J(\mu)=\operatorname{diag}(90,100,70) \mathrm{kg} \mathrm{m}^{2}$, and initial conditions $R(0)=I_{3}, \omega(0)=0$. The reference $\omega_{d}(t)$ has been generated by a neutrally stable linear system with eigenvalues $\lambda=\{ \pm 0.25 i\}$ and initial conditions $\varpi_{d}(0)=$

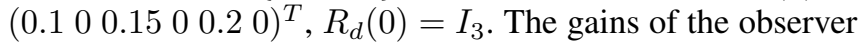
and the tracking controller has been chosen as $k_{1}=40, k_{2}=$ $30, k_{3}=40$, and $\gamma=\nu=2500$. For the internal model, the pairs $\left(F_{i}, G_{i}\right), 1 \leq i \leq 3$, have all been obtained through a balanced realization of a controller companion form with eigenvalues $\lambda=\{-1,-2,-1.5,-2.5\}$, where an initial (incorrect) estimate for the frequencies of the disturbance corresponding to $\theta_{i, 1}=0.5 \mathrm{rad} / \mathrm{s}$ and $\theta_{i, 2}=1.5 \mathrm{rad} / \mathrm{s}, 1 \leq$ $i \leq 3$, was assumed. The initial estimate for $\mu$ has been set to $\hat{\mu}(0)=\left(\begin{array}{lll}120 & 70 & 100\end{array}\right)^{T}$. In all simulations, the adaptation law for the observer is initially disabled, and engaged at $t=$ 50 s.In the first case study, the parameters of the harmonic disturbance have been selected as in Table I, meaning that the exosystem is minimally parameterized by the internal model. The plots in Fig. 1 show the time history of the 2 -norm of the MRP for the observer and the tracking error rotation matrices $\tilde{R}(t)$ and $\hat{R}_{e}(t)$. The top plot and the bottom plot in Fig. 2 show the parameter estimates $\hat{\Psi}_{i}(t)$ and $\hat{\mu}(t)$, respectively. Before the adaptation of the observer is enabled, a sizable tracking error is present and the estimates $\hat{\mu}(t)$ saturate as a consequence of the disturbance. After the adaptation of the observer is complete, regulation is recovered as $\hat{\mu}(t)$ converge to constant values. In this case, not only $\tilde{\Psi}_{i}(t) \rightarrow 0$ as expected, but also $\tilde{\mu}(t) \rightarrow 0$, since the reference trajectory $\boldsymbol{\omega}_{d}(t)$ yields a persistently exciting regressor for $\hat{\mu}(t)$. In the second case study, the PE condition is removed from both the adaptation of the observer and the tracking controller, by setting $a_{1 i}=0,1 \leq i \leq 3$ in the disturbance model, and by choosing the initial condition of the reference command generator as $\varpi_{d}(0)=\left(\begin{array}{llllll}0.1 & 0 & 0 & 0 & 0 & 0\end{array}\right)^{T}$. The rest of the controller parameters have been left unchanged, including the dimension of the internal model. The results of the simulations are presented in Fig. 3. In this case, convergence of $\tilde{\Psi}_{i}(t)$ to the origin has been replaced by convergence onto a lower-dimensional submanifold of the parameter space (see the analysis in [9]).

\begin{tabular}{|l|l|l|}
\hline Ang. vel. & Frequency $\left(\mathrm{rad} / \mathrm{s}^{2}\right)$ & Amplitude $(\mathrm{rad} / \mathrm{s})$ \\
\hline$\omega_{1}$ & $\theta_{11}=1, \theta_{12}=2$ & $a_{11}=0.1, a_{12}=0.15$ \\
\hline$\omega_{2}$ & $\theta_{21}=1, \theta_{22}=3$ & $a_{21}=0.12, a_{22}=0.12$ \\
\hline$\omega_{3}$ & $\theta_{31}=1, \theta_{32}=2.5$ & $a_{31}=0.1, a_{32}=0.1$ \\
\hline
\end{tabular}

TABLE I

Parameters of THE RATE SENSOR Disturbance, CASE STUdy I

\section{CONCLUSIONS}

In this note, a design based on the separation principe has been successfully applied to solve the robust attitude control 

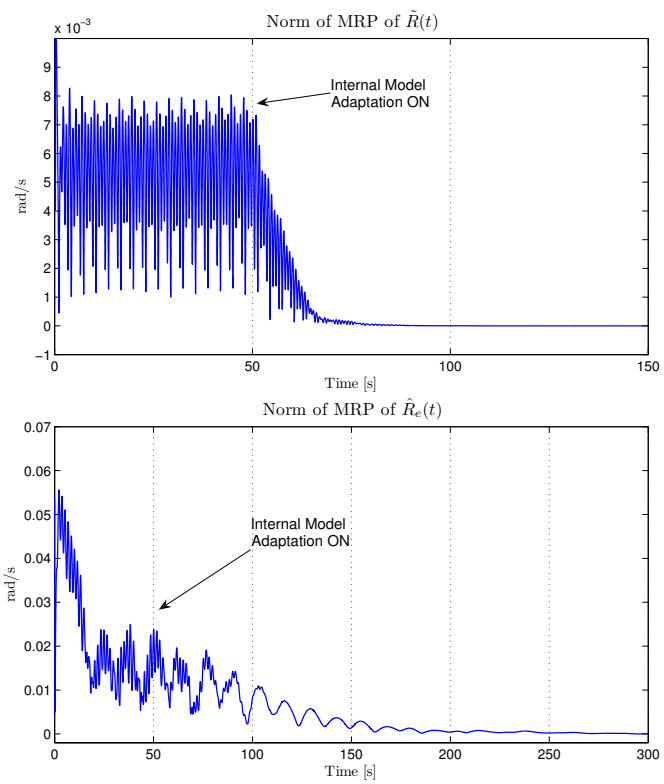

Fig. 1. Norm of $\tilde{\sigma}(t)$ (top) and $\hat{\sigma}_{e}(t)$ (bottom). Case study I.
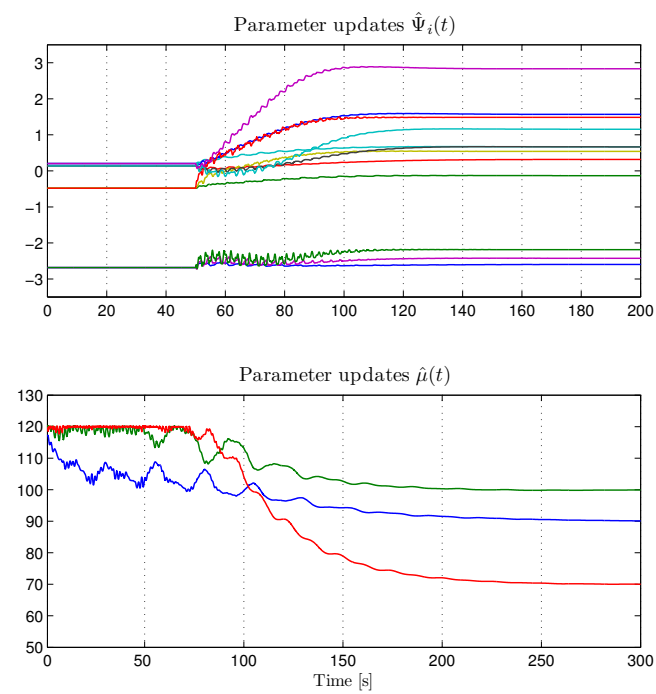

Fig. 2. Parameter updates $\hat{\Psi}_{i}(t)$ (top) and $\hat{\mu}(t)$ (bottom). Case study I.

problem with measurements of the angular rates corrupted by harmonic noise. An adaptive internal model of the disturbance embedded in the observer provides asymptotically accurate estimates of the angular rates, which are employed in a certainty-equivalence passivity-based controller. A very desirable features of the approach lies in the fact that persistence of excitation is not required for adaptive state estimation and error regulation.

\section{REFERENCES}

[1] A. Isidori, L. Marconi, and A. Serrani, Robust Autonomous Guidance: An Internal Model-Based Approach. London, UK: Springer Verlag, 2003.

[2] J. Wen and K. Kreutz-Delgado, "The attitude control problem," IEEE Transactions on Automatic Control, vol. 36, no. 10, pp. 1148-1162, 1991.
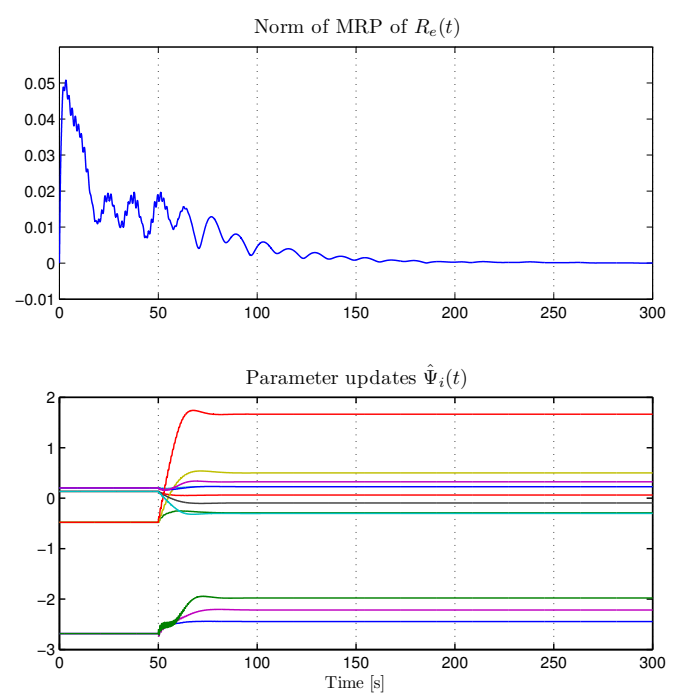

Fig. 3. Norm of $\sigma_{e}(t)$ (top) and parameters $\hat{\Psi}_{i}(t)$ (bottom). Case study II.

[3] F. Chavez and D. Schmidt, "Uncertainty modeling for multivariablecontrol robustness analysis of elastic high-speed vehicles," Journal of Guidance, Control, and Dynamics, vol. 22, no. 1, pp. 87-95, 1999.

[4] C. J. Heiberg, D. Bailey, and B. Wie, "Precision spacecraft pointing using single-gimbal control moment gyroscopes with disturbance," Journal of Guidance, Control, and Dynamics, vol. 23, no. 1, pp. 7785,2000

[5] B. Li, D. Hullender, and M. DiRenzo, "Nonlinear induced disturbance rejection in inertial stabilization systems," IEEE Transactions on Control Systems Technology, vol. 6, no. 3, pp. 421-7, 1998.

[6] J. Thienel and R. Sanner, "A coupled nonlinear spacecraft attitude controller and observer with an unknown constant gyro bias and gyro noise," IEEE Transactions on Automatic Control, vol. 48, no. 11, pp. 2011-2015, 2003.

[7] M. Bolender and D. Doman, "Modeling unsteady heating effects on the structural dynamics of a hypersonic vehicle," in Proceedings of the AIAA Guidance, Navigation, and Control Conference and Exhibit, Keystone, CO, 2006, AIAA Paper 2006-6646.

[8] P. Pisu, A. Serrani, and G. Rizzoni, "Rejection of harmonic sensor disturbances in passive systems, and applications to attitude control," in Proceedings of the American Control Conference, Minneapolis, MN, 2006.

[9] A. Serrani, "Rejection of harmonic disturbances at the controller input via hybrid adaptive external models," Automatica, vol. 42, no. 11, pp. 1977-1985, 2006.

[10] O. Egeland and J.-M. Godhavn, "Passivity-based adaptive attitude control of a rigid spacecraft," IEEE Transactions on Automatic Control,, vol. 39, no. 4, pp. 842-846, April 1994.

[11] A. Serrani, A. Isidori, and L. Marconi, "Semi-global nonlinear output regulation with adaptive internal model," IEEE Transactions on Automatic Control, vol. 46, no. 8, pp. 1178-1194, 2001.

[12] B. Vik, A. Shiriaev, and T. Fossen, "Nonlinear observer design for integration of DGPS and INS," in New Directions in Nonlinear Observer Design, ser. LNCIS, Vol. 244, H. Nijmeijer and T. Fossen, Eds. Springer-Verlag, 1999, pp. 135-159.

[13] P. Tsiotras, "Stabilization and optimality results for the attitude control problem," Journal of Guidance, Control, and Dynamics, vol. 19, no. 4, pp. 772-779, 1996.

[14] M. Krstić, I. Kanellakopoulos, and P. Kokotović, Nonlinear and Adaptive Control Systems. New York, NY: John Wiley and sons, 1995. 